\title{
Evaluación de infraestructura mediante accesibilidad territorial. Caso de estudio: conexión Villamaría-Manizales (Colombia)
}

\begin{abstract}
Diego A. Escobar(1)*, Santiago Cardona(1) y Carlos A. Moncada(2)
(1) Universidad Nacional de Colombia - Sede Manizales. Facultad de Ingeniería y Arquitectura, Departamento de Ingeniería Civil, Grupo de Investigación en Movilidad Sostenible, Campus La Nubia, Bloque S2-208, Carrera 37 con Calle 94, Manizales, 170003. Colombia. (correo-e: daescobarga@unal.edu.co; scardonau@unal.edu.co)

(2) Universidad Nacional de Colombia - Sede Bogotá. Facultad de Ingeniería, Departamento de Ingeniería Civil y Agrícola, Programa de Investigación en Tránsito y Transporte - PIT. Ciudad Universitaria edificio 214 oficina 417, Bogotá, 111321. Colombia. (correo-e: camoncadaa@unal.edu.co)
\end{abstract}

* Autor a quien debe ser dirigida la correspondencia.

Recibido Ago. 5, 2019; Aceptado Sep. 30, 2019; Versión final Oct. 22, 2019, Publicado Jun. 2020

\section{Resumen}

El objetivo de la presente investigación consiste en evaluar la mejor alternativa para la segunda conexión de acceso entre la ciudad de Manizales y el municipio de Villamaría, bajo la necesidad de mejorar las condiciones del flujo vehicular, incrementado por la condición de conurbación existente. La metodología de estudio emplea la accesibilidad territorial, de carácter global, como elemento de evaluación de impacto, a través del cálculo y cuantificación del ahorro generado en los tiempos promedio de viaje, tomando como base el análisis de la conexión actual. Como principal resultado se obtiene que las intervenciones propuestas, generan un impacto positivo entre ambas municipalidades, generando ahorros de tiempo del $22 \%$ en el tiempo de viaje. Como conclusión principal, se puede argumentar que el uso de la accesibilidad territorial permite evaluar de forma eficaz, la mejor opción de intervención en una etapa de pre factibilidad en proyectos de inversión en transporte. vial; movilidad

\section{Infrastructure evaluation through territorial accessibility. Case Study: Villamaría-Manizales connection (Colombia)}

\begin{abstract}
The objective of this research was to evaluate the best alternative for a second access connection to improve traffic flow conditions between the city of Manizales and the municipality of Villamaría. The methodology used territorial accessibility of global nature as an impact evaluation element. This was performed by quantifying the savings generated in average travel times when compared to that of the existing connection (basis). The proposed intervention on the present study generates a positive impact for both municipalities by generating time savings of up to $22 \%$ in travel time. It can be argued that using territorial accessibility allows efficient evaluation of the best intervention option for transport investment projects in a pre-feasibility stage.
\end{abstract}




\section{INTRODUCCIÓN}

En los últimos años, se ha visto el interés por la incorporación de modelos de transportes en la toma de decisiones para las intervenciones en infraestructura como apertura de vías, implementación de puentes, intersecciones, al igual que en la implementación de sistemas de transporte como metro, BRT, cable aéreo, tranvías, debido al alto costo en parámetros fiscales, monetarios y tiempos, desde su planeación y construcción su puesta en funcionamiento (Marvin y Guy, 1999; Ortúzar y Willumsen, 1994; Te Brömmelstroet y Bertolini, 2011). En este sentido, los modelos basados en la accesibilidad, buscan cuantificar la relación existente entre la oferta del transporte y la localización de los equipamientos y la distribución de la población, con el fin de establecer parámetros operativos actuales y seleccionar la mejor alternativa de intervención basados en el mayor beneficio, son estos los llamados modelos de usos del suelo y transporte (Cerda et al., 2012a). Las diferentes instituciones gubernamentales han adoptado diversas metodologías, en búsqueda de organizar de la mejor manera el planeamiento de las infraestructuras, por ejemplo, en Colombia, a través de la ley 338 de 1997 se reglamentó la elaboración de los Planes de Ordenamiento Territorial (POT), en el ámbito del urbanismo, los cuales funcionan como herramienta de planificación y ordenamiento del territorio estableciendo reglas en múltiples áreas como usos del suelo, medio ambiente, construcción, entre otros; éstos instrumentos de planificación definen diferentes alcances, según el número de habitantes de la ciudad o municipio y poseen una vigencia de doce (12) años. No obstante, como instrumento de planificación, aplicado en diferentes municipios de Colombia, se ha identificado una desarticulación acentuada entre el ordenamiento y los planes de movilidad y transporte, haciendo de lado los planteamientos cuantitativos que puedan ofrecer una visión territorial e integrada (Cerda et al., 2012a).

De igual forma, mediante la ley 1083 de 2006, se establecieron las acciones que se deben llevar a cabo en el ámbito de la movilidad urbana en las ciudades, en búsqueda de la sostenibilidad en el transporte, priorizando los modos alternativos como la bicicleta y el transporte público colectivo, así como la migración a tecnologías más limpias y eficientes. Dichas acciones deben ser ejecutadas en el marco de un Plan de Movilidad, mediante el diagnóstico de la situación actual y la formulación de los proyectos requeridos para solventar los problemas de movilidad en periodos de corto, medio y largo plazo. Con respecto a esto, Manizales, capital del departamento de Caldas (figura 1) ejecutó y aprobó en el año 2017, el Plan de Ordenamiento Territorial 2017 - 2031 (Alcaldía de Manizales, 2017a) y el Plan Maestro de Movilidad 2017 - 2032 - PMM (Alcaldía de Manizales, 2017b).
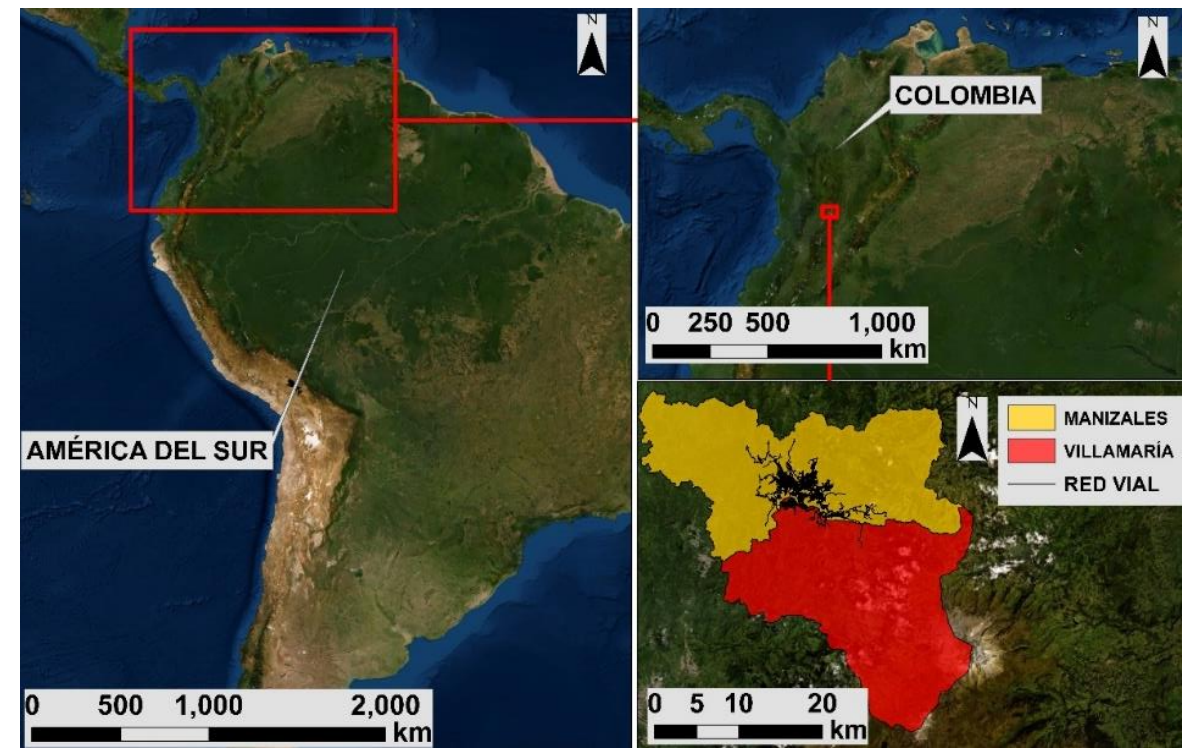

Fig 1: Localización de la zona de estudio (Adaptada de Esri, DigitalGlobe, GeoEye, Earthstar Geographics, CNES/Airbus DS, USDA, USGS, AeroGRID, IGN, y tha GIS User Community)

Manizales cuenta con un total de 982.231 viajes diarios, con un amplio porcentaje de usuarios que se movilizan a pie $(29 \%)$ y en transporte público $(24 \%)$, el cual posee ofertas flexibles cómo bus, buseta, cable aéreo y colectivo, vehículo privado $(25 \%)$ con una tasa de motorización creciente, traduciéndose esto en una insostenibilidad de la red de infraestructuras del transporte en las próximas décadas. Lo anterior resalta la importancia de las acciones propuestas en el PMM (2017) a través de los 138 proyectos desarrollados a lo largo de 6 pilares cómo la potenciación el espacio libre de tránsito peatonal, el uso de la bicicleta y el desarrollo del Sistema Estratégico de Transporte Publico- SETP, entre otras. Dentro de estos proyectos, se incluyen las diversas propuestas para la estructuración de un segundo acceso vial al municipio de Villamaría, debido a la condición actual de acceso, la cual suple sus desplazamientos sobre una única conexión, generando fuertes 
problemáticas de congestión, incrementando los tiempos de viaje de los usuarios para los múltiples viajes que se realizan entre ambos municipios; considerados una conurbación con una población de 419.000 habitantes para 2017 aproximadamente (DANE, 2010). Teniendo en cuenta lo anterior, el PMM propone tres alternativas futuras de conexión para Villamaría (ver figura 2), las cuales serán evaluadas en esta investigación a partir del uso de la accesibilidad territorial, estimando la cobertura poblacional por las nuevas obras de infraestructura (Escobar et al., 2018; Martínez et al., 2017; Moncada et al., 2018).

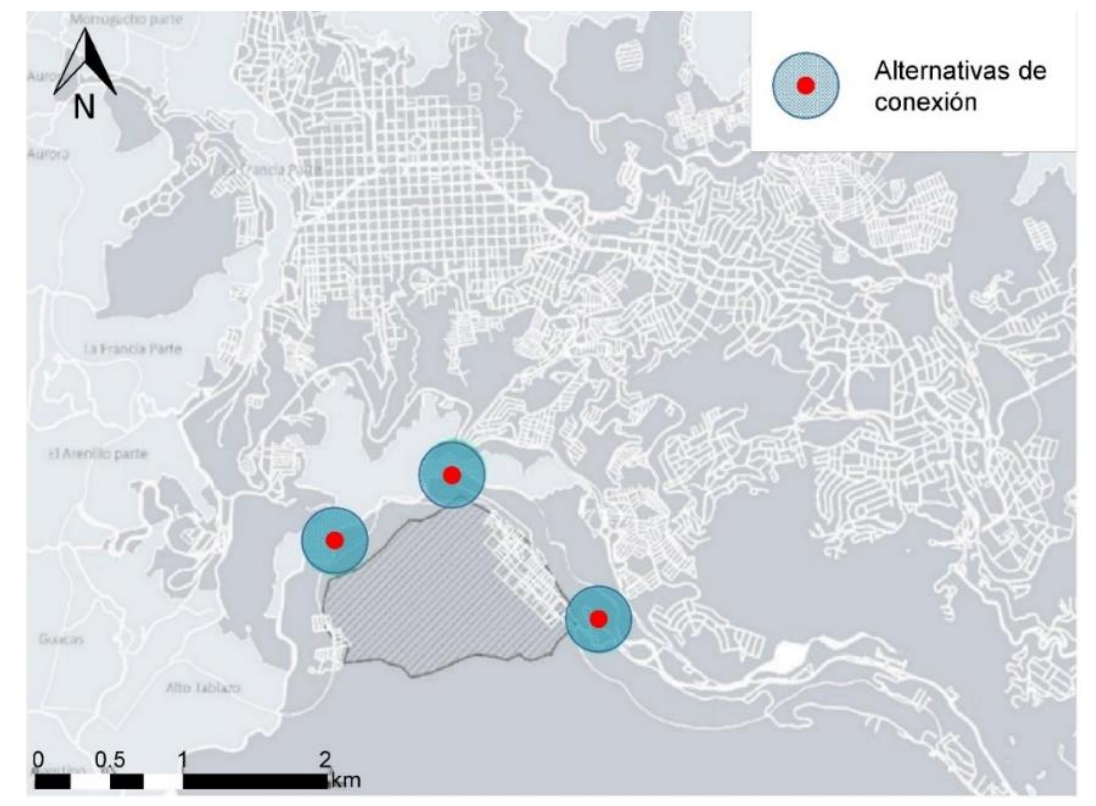

Fig. 2: Alternativas de conexión Villamaría - Manizales (Adaptada de Alcaldía de Manizales, 2017b).

La accesibilidad territorial de carácter global, se establece como un modelo de oferta del transporte, sobre el cual se determina el grado de conexión entre todos los nodos de una red vial mediante el uso del algoritmo de caminos mínimos (Dijkstra, 1959), sobre el cual se calcula la matriz de tiempos medios de viaje. Sin embargo, este concepto ha mutado a lo largo de los años, tomando como origen la definición de Hansen (1959) “... the potencial opportunities for interaction...", y derivando en múltiples enfoques (Ingram, 1971) y medidas (Pirie, 1979). Así mismo, a través de los años, diversos investigadores han realizado revisiones del concepto (Dalvi y Martin, 1976; Geurs y Van Eck, 2001; Morris et al., 1979), definiendo además el enfoque futuro y los distintos desafíos que posee en el campo investigativo y de planeamiento urbano (Geurs, et al., 2015; Van Wee, 2016). Por otro lado, fue Weibull quién en 1976 definió la accesibilidad como la proximidad física entre dos puntos geográficos y además propuso los principales axiomas que se deben cumplir para realizar un cálculo acertado de la medida. La accesibilidad media global se basa en la infraestructura existente, según las perspectivas adoptadas por Geurs y Van Eck (2001) ya que utiliza la red de infraestructura vial y los parámetros operativos (velocidad, direccionalidad, tipología, etc.) como insumo fundamental para el cálculo (Moncada et al., 2018). En la presente investigación se comparan y evalúan cuatro alternativas de conexión infraestructural (Dos alternativas en el Oeste, una en el Norte y una al Este) entre las poblaciones contiguas de Manizales y Villamaría, evaluación que se realiza en base al análisis de la variable accesibilidad y cobertura poblacional según el estrato socioeconómico. Existen otros análisis que podrían complementar la presente investigación, tomando como base modelos cómo el EVALUAMET el cual incluye nuevas variables a los modelos de transportes, por ejemplo, variables de tipo ambiental y de eficiencia energética (Cerda et al., 2012a), en el cual se reconocen y combinan tres enfoques de evaluación: la cuantificación del consumo energético ambiental, la cuantificación del consumo del suelo, y la evaluación social de la equidad en el acceso al territorio. Así mismo, existen investigaciones que buscan evaluar el impacto de los proyectos infraestructurales y/o urbanísticos, los cuales producen efectos diferenciales en las dimensiones sociales y de actividades económicas y su interacción (Cerda et al., 2012b).

Todo lo anterior, pone de manifiesto que los desafíos a los cuales se enfrenta la formulación de políticas públicas, pasa por involucrar de forma decidida y concreta, criterios de sostenibilidad, tanto ambiental como social para la evaluación y correcta puesta en marcha de estrategias, planes y programas de intervención infraestructural, en los cuales se observe una verdadera interacción entre la accesibilidad o modelos de transporte y el crecimiento urbano, para lo cual la presente investigación se considera como un importante punto de partida, ya que conocer con claridad la relación entre dichos enfoques, es fundamental para el diseño de políticas urbanas. Luego de esta introducción, se expone la Metodología de investigación, los principales resultados y discusión de los mismos, así como las conclusiones finales de la investigación. 


\section{METODOLOGÍA}

En la figura 3 se presenta el modelo metodológico empleado para el desarrollo de la investigación; este modelo cuenta con un total de 5 etapas consecutivas que se describen a continuación.

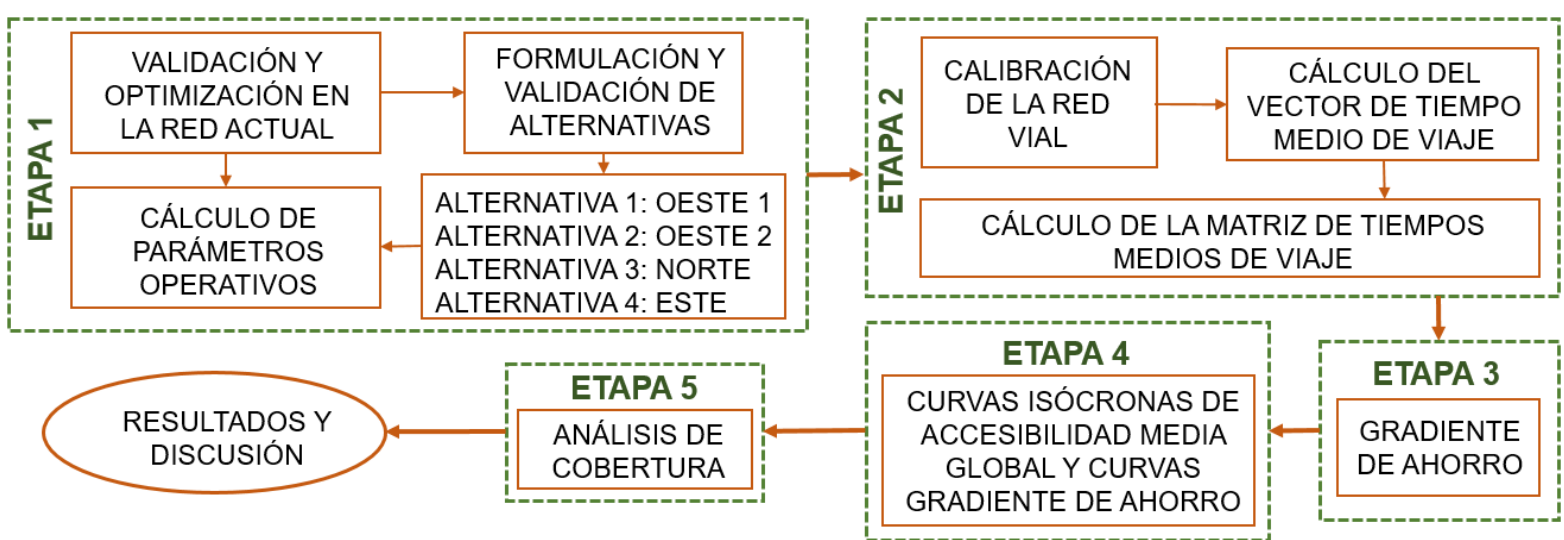

Fig. 3: Metodología de investigación abordada

Validación y optimización de la red vial actual y alternativas propuestas.

En primer lugar, se incurre en la adquisición de la red de infraestructura vial urbana de Manizales y Villamaría; posteriormente se realiza la validación de la red, garantizando el óptimo funcionamiento de los parámetros físicos y operativos asociados, mediante la adecuada aplicación de la teoría de grafos, en la cual se considera que, un grafo $(G)$ se compone por un conjunto de vértices $(V)$ (nodos de conexión vial) y aristas (E) (arcos o links de desplazamiento), que permiten la interpretación o abstracción de la realidad (Xiao, 2016; De Smith et al., 2018). La red de infraestructuras del transporte empleada proviene de estudios asociados al PMM (Alcaldía de Manizales, 2011), información oficialmente disponible y provista directamente por la Alcaldía municipal, la cual ha sido actualizada constantemente en investigaciones recientes, incluyendo obras de infraestructura vial actualmente operativas (Martínez et al., 2017; Moncada et al., 2018; Montoya et al., 2017); la red de infraestructura vial con la que se realizaron los cálculos está compuesta por 10.100 nodos que conectan 12.732 arcos, topológicamente es del tipo malla ya que todos los nodos se conectan de tal forma que no existe una preeminencia de un nodo sobre otros, es así que teniendo en cuenta los sentidos viales de la misma, proporcionan continuidad y conectividad. En segundo lugar, se realiza la formulación y validación de las cuatro (4) alternativas de conexión a evaluar, según las propuestas establecidas en el PMM del 2017 (ver figura 4), incluyéndolas en el grafo de la red de infraestructuras del transporte. La alternativa Oeste 1 conecta al barrio la pradera de Villamaría, con la Cárcel Nacional de Varones en Manizales; la alternativa Oeste 2 conecta el barrio la Pradera de Villamaría con el sector de la Uribe en Manizales; la alternativa Norte sería la conexión de Villamaría hacia la intersección vial de la Fuente en Manizales; y la alternativa Este conecta a Villamaría con el terminal de transportes, siendo esta la propuesta más cercana a la conexión actual.

\section{Calibración de la red de infraestructura vial y cálculo del vector de tiempo medio de viaje}

Tanto en la situación actual cómo en las alternativas propuestas, se calculan los tiempos de viaje (tvi) por arco, teniendo en cuenta su longitud (li) calculada en ArcGis y su velocidad operativa (Vi). La variable velocidad operativa se calculó mediante la instalación de cincuenta dispositivos GPS en diferentes vehículos automotores (carro, taxi, motocicleta, camión y buses), mediante los cuales se obtuvo información de su posición geográfica (longitud, latitud) en intervalos de 10 segundos, a lo largo de un año (2017). Se recopilaron más de 18 millones de datos. Los valores calculados de velocidad operativa varían entre $5 \mathrm{~km} / \mathrm{h}$ en zonas del centro histórico de la ciudad y hasta $80 \mathrm{~km} / \mathrm{h}$ sobre arcos de algunas de las vías arterias principales, encontrando que los resultados obtenidos son estadísticamente representativos. Para integrar estas velocidades al grafo de la red vial se utilizó un software desarrollado por el grupo de investigación en movilidad sostenible, complementando los arcos sin información según la jerarquía de la vía propuesta en el PMM del año 2017 (Alcaldía de Manizales, 2017b). Para cada alternativa propuesta, así como para la situación actual, se calculó el vector de tiempo medio de viaje, mediante el uso del software TRANSCAD 7.0, el cual es una herramienta especializada en el campo transporte. En primera instancia se obtuvo la matriz de tiempos medios de viaje entre todos los nodos que componen la red de infraestructura vial, a partir del algoritmo de Dijkstra o algoritmo de caminos mínimos (Dijkstra, 1959; Xiao, 2016; De Smith et al., 2018), el cual optimiza el tiempo medio de viaje entre los 10.100 nodos que conectan 12.732 arcos que, en conjunto, componen la red de infraestructura vial. 


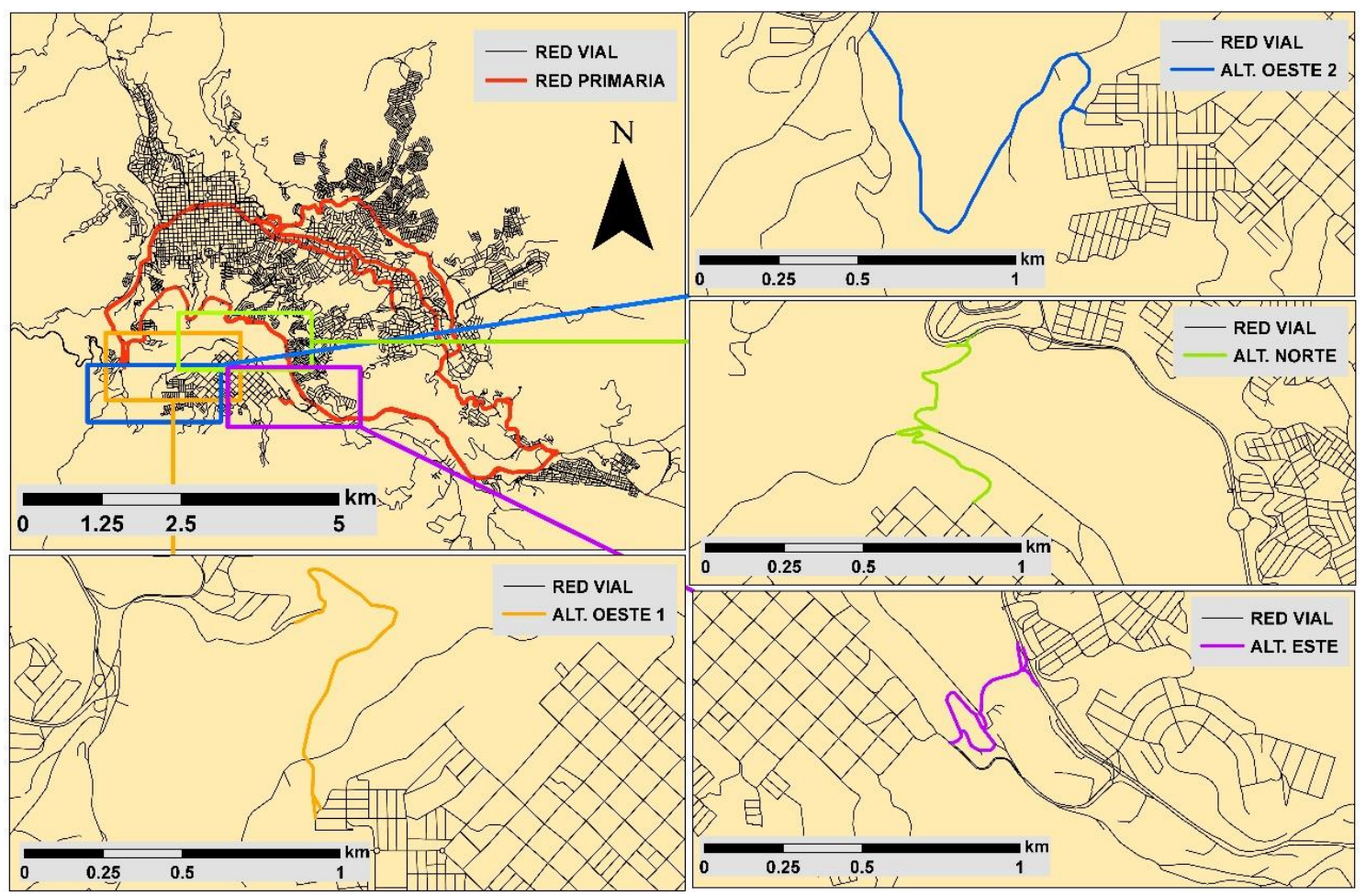

Fig. 4: Escenario actual y las alternativas de conexión analizadas.

Para la calibración de la red se tuvieron en cuenta las penalidades por giro, calculadas mediante un trabajo de campo que registro el tiempo de giro en intersecciones simples y semaforizadas, obteniendo cómo valores de 0,58 minutos a la izquierda y 0,49 minutos a la derecha (Cardona, 2018). Luego de obtener la matriz de tiempos de viaje, se procede a calcular el vector de tiempo promedio de viaje $\mathrm{TV}_{\mathrm{ij}}$, mediante la ecuación (1), donde se tiene en cuenta la sumatoria de tiempo de viaje entre los pares de nodo origen (i) y destino (j) y el total de nodos a los cuales se puede viajar en la red (n-1) (Escobar et al., 2018; Moncada et al., 2018). Así mismo, este vector de tiempos promedio de viaje debe relacionarse con las coordenadas geográficas de cada nodo, con lo cual se efectúa el análisis geoestadístico en etapas posteriores.

$$
\overline{\mathrm{Tvl}}=\frac{\sum_{\mathrm{j}=1}^{\mathrm{n}} \mathrm{tv}_{\mathrm{ij}}}{\mathrm{n}-1}
$$

Este método de cálculo de la velocidad operativa y del vector de tiempo medio de viaje, mediante equipos GPS incluye implícitamente las condiciones de congestión que se presentan en diferentes horarios, días y zonas de la ciudad. Por otra parte, en el caso de las alternativas de conexión propuestas, se definió una velocidad promedio de $45 \mathrm{~km} / \mathrm{h}$, siendo está la velocidad de operación esperada.

\section{Gradiente de ahorro}

Una vez obtenidos los tiempos de viaje para cada escenario, se procede a determinar el vector de gradiente de ahorro $V_{s g}(\%)$ donde se compara la situación actual de accesibilidad media global con los cuatro (4) escenarios propuestos de segunda conexión entre Villamaría y Manizales. Para esto, se utiliza la ecuación 2, donde ( $\left.T v_{c s}\right)$ corresponde al vector de tiempo promedio de viaje de la situación actual y ( $\left.T v_{p s}\right)$ es el vector de tiempo promedio de viaje de los escenarios propuestos (Moncada et al., 2018). En este caso se calculan cuatro vectores de gradiente de ahorro que corresponde al porcentaje ahorrado a los tiempos de viaje respecto a la situación actual generado por las nuevas alternativas de conexión entre Villamaría y Manizales.

$$
\mathrm{V}_{\mathrm{sg}}(\%)=\left(\frac{\overline{\mathrm{Tv}_{\mathrm{cs}}}-\overline{\mathrm{Tv}_{\mathrm{pss}}}}{\overline{\mathrm{Tv}_{\mathrm{cs}}}}\right) * 100 \quad \mathrm{i}=1,2,3,4
$$

\section{Curvas isócronas de accesibilidad media global y curvas gradiente de ahorro}

Para la construcción de las curvas isócronas de accesibilidad media global y de gradiente ahorro, en cada escenario analizado, se aplica la geoestadística, cuyo principal uso es el de predecir valores de un atributo en un sitio específico, en este caso, tiempo medio de viaje, a partir de valores conocidos del mismo atributo 
en otros puntos, en otras palabras, interpolación (Chun y Griffith, 2013). Para el cálculo geoestadístico se usó la herramienta Geostatical Wizard de ArcMAp, la cual incluye el uso del modelo geo estadístico Kriging ordinario, con semivariograma lineal como ecuación estructural (Chun y Griffith, 2013; Díaz, 2002; Oliver y Webster, 1990), este modelo ha sido utilizado en diversas investigaciones en temas relacionados con el transporte (Escobar et al., 2018; Zhang y Wang, 2014) y es conocido en el ámbito investigativo por una predicción optima y minimizar la varianza del error, siendo considerado como el mejor predictor lineal imparcial (Chun y Griffith, 2013; Xiao, 2016). En este caso, como insumo de interpolación se utilizan los vectores de tiempo promedio de viaje y los vectores de gradiente de ahorro para la construcción de curvas.

\section{Análisis de cobertura}

Finalmente, se realiza el análisis de cobertura poblacional a partir de las curvas isócronas de tiempo medio de viaje y ahorro obtenidas; superponiendo el shape de barrios de la zona de estudio (Moncada et al., 2018), el cual posee información referente a la población residente y el estrato socioeconómico al cual pertenece. Dicha información demográfica es la que oficialmente el Departamento Administrativo Nacional de Estadística ha proyectado para el año 2017 (DANE, 2010). La población fue distribuida en las nuevas áreas mediante el método de áreas proporcionales que puede aplicarse mediante el SIG. El estrato socioeconómico se define como una medida diferencial de los usuarios residentes a partir de la capacidad económica, siendo 1 (baja) la valoración mínima y 6 (alto) los residentes con el mayor ingreso (Martínez et al, 2018). A partir de esta información, es posible obtener la ojiva porcentual de población cubierta (Xiao, 2016; De Smith et al., 2018) en el caso de la accesibilidad media global y la gráfica tipo "Elbow" de cobertura de ahorro en el caso del gradiente de ahorro (Moncada et al., 2018).

\section{RESULTADOS Y DISCUSIÓN}

Para el escenario actual, la conexión vial existente entre Villamaría y Manizales cuenta con una vía doble carril que se enlaza mediante túnel a la vía panamericana, en cercanías al terminal de transportes de la ciudad. Esta conexión, al ser única, posee alta congestión vehicular lo que generará un colapso en la movilidad entre estas dos conurbaciones en los próximos años (Alcaldía de Manizales, 2017b). En cuanto a las alternativas de conexión propuestas, el PMM del año 2017, señaló tres diferentes lugares de posible ubicación (ver figura 2). Ahora bien, en la figura 5 , se observa el comportamiento del tiempo medio de viaje para el escenario actual en relación con la conexión entre Villamaría y Manizales.

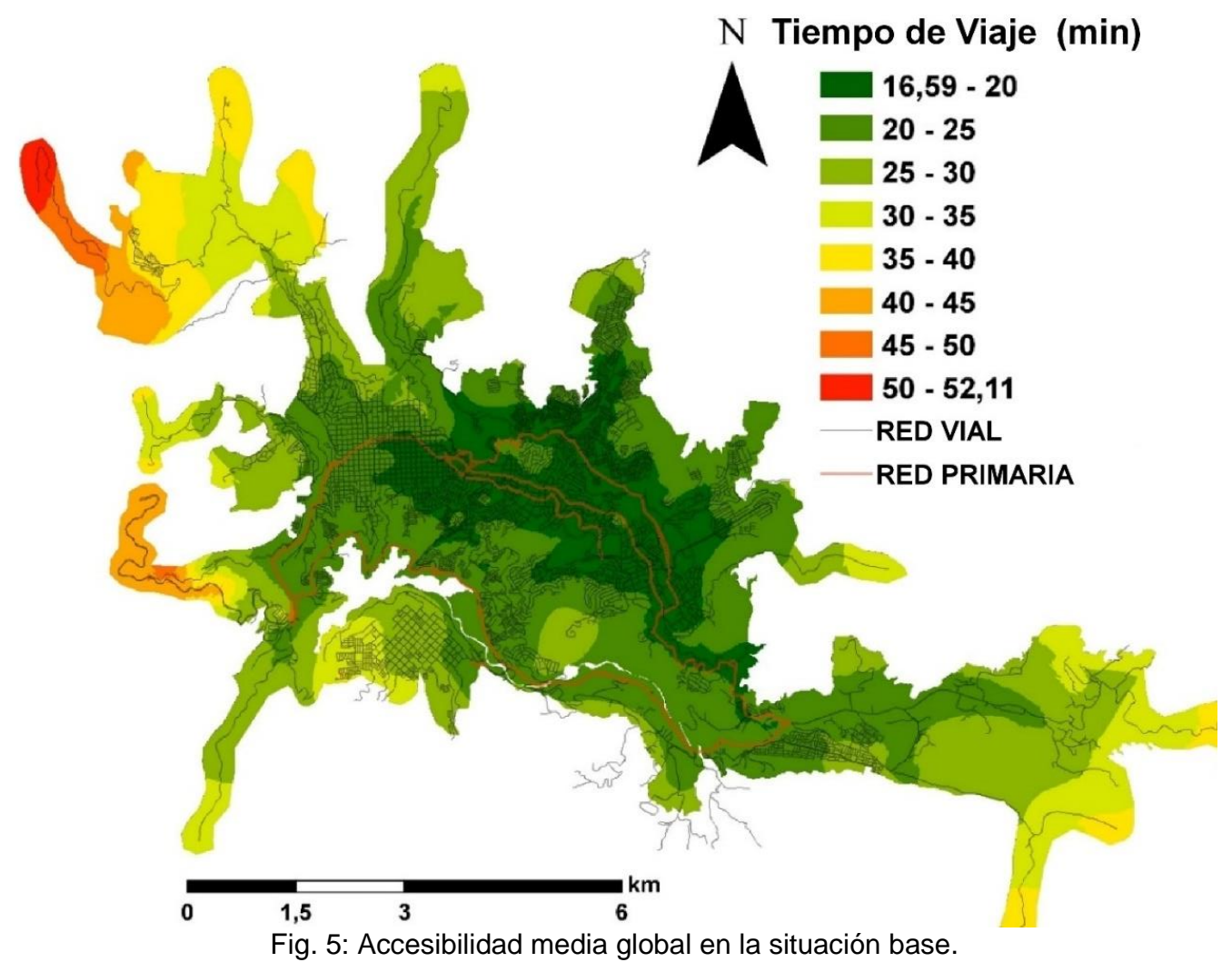

Los resultados presentan ciertas similitudes respectos a estudios anteriores (Escobar et al., 2018; Cardona, 2018); sin embargo, se registran tiempos mínimos de 16,59 minutos y máximos 52,11 minutos. En la parte central de la ciudad se observa una cobertura eficiente, con tiempos promedio de viaje menores a 25 minutos, 
traduciendo una buena accesibilidad para los usuarios que se desplazan desde este sector a través de la red de infraestructura vial. Por otro lado, en las partes periféricas de la ciudad, se registran tiempos superiores a 40 minutos, que alcanzan los 52,11 minutos en la parte noroeste de la ciudad. En la tabla 1, se observa el análisis realizado entre las curvas isócronas y la cobertura de población, en donde, para la situación actual se tuvo como resultado que el $81,8 \%$ de la población (208.932 habitantes) logra ser cubierta en un tiempo promedio de viaje de hasta 25 minutos. De igual forma, para los escenarios propuestos, se observa un comportamiento similar en cobertura; en donde las alternativas Norte y Oeste 2 aumentaron la cobertura en un $4 \%$, la alternativa Oeste 1 aumento este porcentaje en 2,5\%, por último, el escenario Este presenta el aumento de cobertura más bajo con un $0,2 \%$, para el mismo tiempo medio de viaje de 25 minutos.

Tabla 1. Porcentaje de cobertura poblacional Vs Accesibilidad media global.

\begin{tabular}{|c|c|c|c|c|c|}
\hline $\begin{array}{c}\text { Curvas } \\
\text { Isócronas } \\
(\mathrm{min})\end{array}$ & $\begin{array}{c}\text { Escenario } \\
\text { Actual }\end{array}$ & $\begin{array}{c}\text { Alternativa } \\
\text { Oeste 1 }\end{array}$ & $\begin{array}{c}\text { Alternativa } \\
\text { Oeste 2 }\end{array}$ & $\begin{array}{c}\text { Alternativa } \\
\text { Este }\end{array}$ & $\begin{array}{c}\text { Alternativa } \\
\text { Norte }\end{array}$ \\
\hline $16,59-20$ & $32,0 \%$ & $34,1 \%$ & $35,3 \%$ & $32,0 \%$ & $36,2 \%$ \\
\hline $20-25$ & $81,8 \%$ & $84,3 \%$ & $85,7 \%$ & $82,0 \%$ & $85,8 \%$ \\
\hline $25-30$ & $95,4 \%$ & $98,7 \%$ & $98,9 \%$ & $95,5 \%$ & $98,4 \%$ \\
\hline $30-35$ & $99,0 \%$ & $99,1 \%$ & $99,1 \%$ & $99,0 \%$ & $99,1 \%$ \\
\hline $35-40$ & $99,7 \%$ & $99,8 \%$ & $99,8 \%$ & $99,7 \%$ & $99,8 \%$ \\
\hline $40-52,11$ & $100,0 \%$ & $100,0 \%$ & $100,00 \%$ & $100,00 \%$ & $100,0 \%$ \\
\hline
\end{tabular}

En la figura 6 se observa el resultado de las curvas isócronas de accesibilidad media global para las diferentes alternativas estudiadas. Entre los resultados más relevantes se tiene que la alternativa Oeste 1 disminuyó el tiempo promedio de viaje mínimo en 0,82 minutos, mientras que las demás alternativas lo disminuyeron en aproximadamente de 0,20 minutos e incluso, la alternativa Este no registró cambios significativos ya que tan solo disminuyó el tiempo medio de viaje en 0,01 minutos.

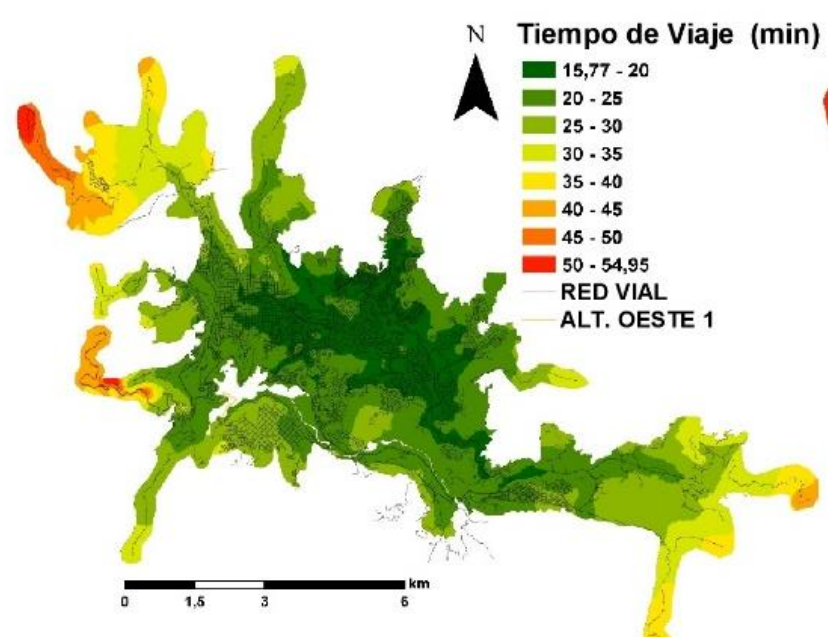

a

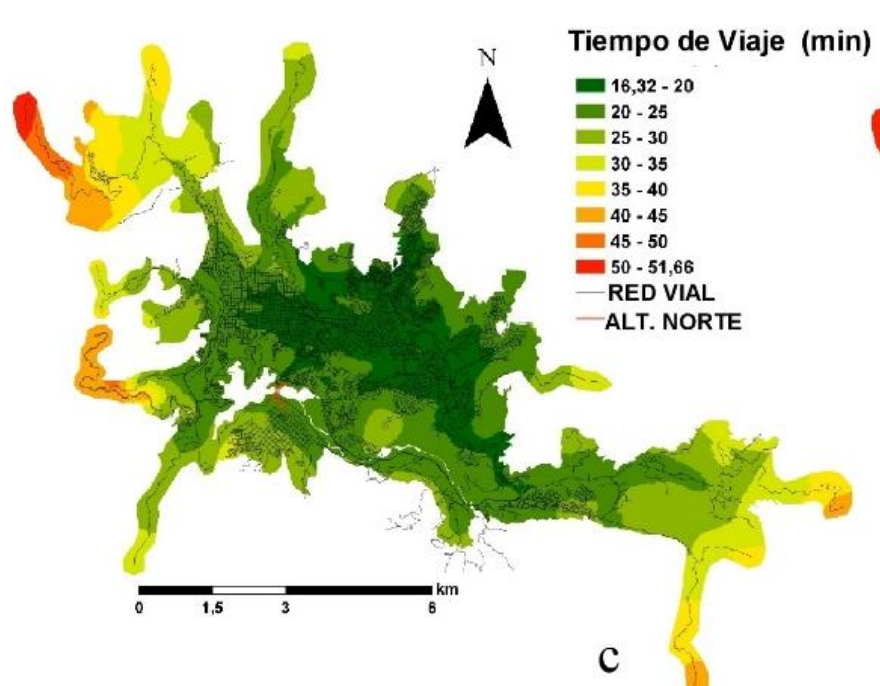

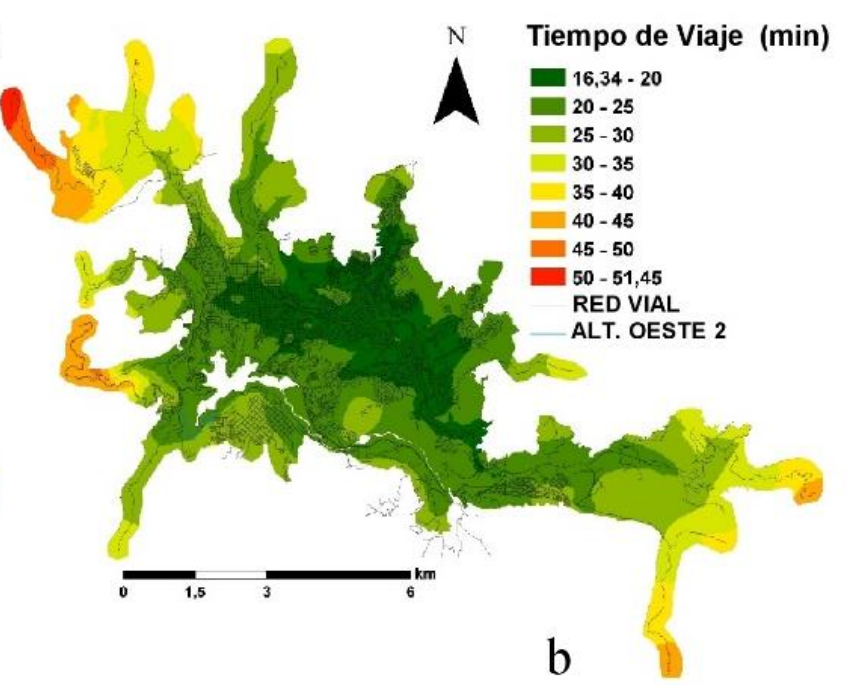

b

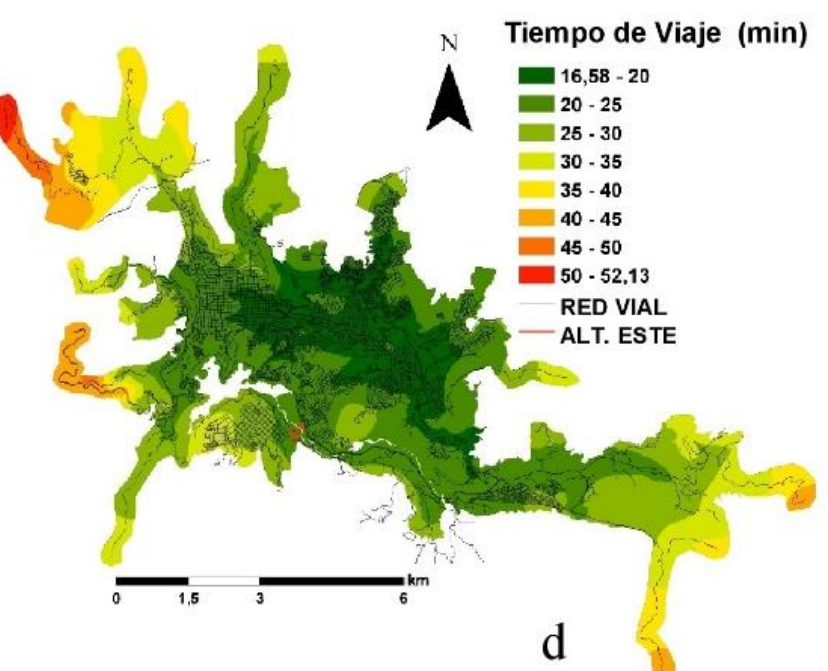

Fig. 6: Accesibilidad media global. a) Alternativa Oeste 1; b) Alternativa Oeste 2; c) Alternativa Norte; d) Alternativa Este. 
Por otra parte, como resultado del análisis de ahorro en el tiempo medio de viaje, se obtiene la figura 7 , donde se presenta el comportamiento de las curvas gradiente de ahorro para las cuatro alternativas en relación con el escenario actual; se observan ahorros de hasta el $30 \%$ en la alternativa Oeste 2 . Asimismo, la alternativa Oeste 1 y Norte generan ahorros en la población de hasta el 22,5\%, mientras que la alternativa Este tan solo alcanza ahorros de hasta el 7,5\%, siendo esta la alternativa menos eficiente.

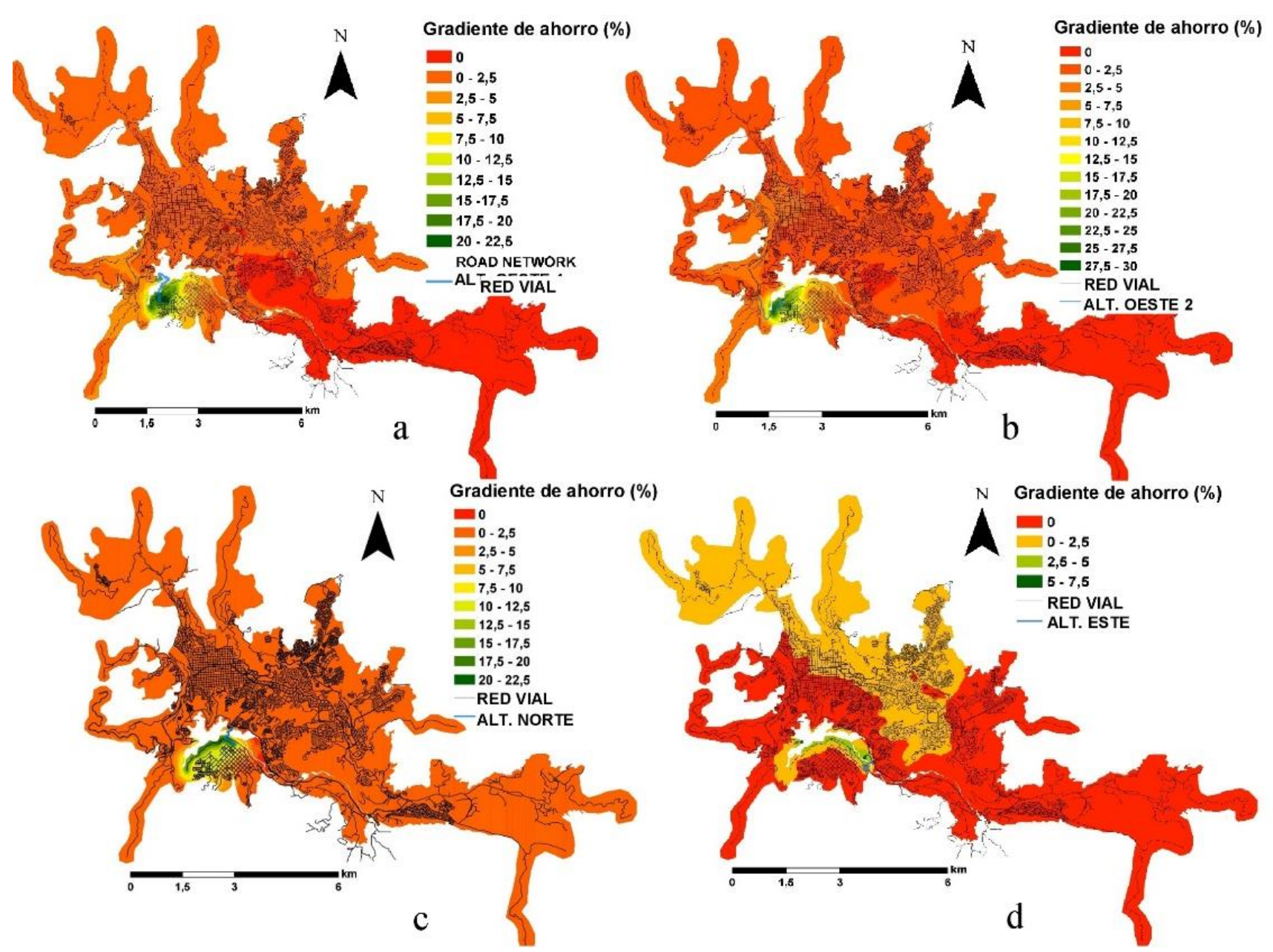

Fig. 7: Gradiente de Ahorro en tiempo medio de viaje en: a) Alternativa Oeste 1; b) Alternativa Oeste 2; c) Alternativa Norte; d) Alternativa Este.

Al realizar un cálculo del porcentaje de ahorro ponderado por la cantidad de población cubierta por cada curva gradiente, se obtiene que la alternativa Oeste 2 registra un ahorro del 3,3\% en relación con el tiempo medio de viaje del escenario actual, seguida de la alternativa Norte con un 3,2\%, la alternativa Oeste 1 con un 2,9\% y finalmente la alternativa Este con un 1,2\%. Por otro lado, aunque la alternativa Oeste 2 genera el mayor porcentaje de ahorro, éste no se presenta sobre la totalidad de la zona de estudio, mientras que la alternativa Norte proporciona un ahorro de hasta el 2,5\% para el 99,5\% de la población (Ver Tabla 2); para el mismo rango de ahorro de porcentaje de ahorro de tiempo medio de viaje, las alternativas Oeste 1, Oeste 2 y Este las cuales alcanzan a cubrir porcentajes de población de $86,3 \%, 73,0 \%$ y $46,2 \%$, respectivamente. Teniendo en cuenta ahorros de hasta el $5 \%$ de tiempo medio de viaje, la alternativa Oeste 2 alcanza a cubrir $12,8 \%$, seguido por las alternativas Oeste 1 y Norte, que alcanzan porcentajes de población de $10,0 \%$ y $9,4 \%$, respectivamente; en este intervalo de ahorro de tiempo medio de viaje, la alternativa Este tan solo alcanza a cubrir el 1,3\% de población demostrando los pocos beneficios de su construcción en términos de accesibilidad media global; asimismo, no genera porcentajes de ahorro en los demás intervalos mientras que las demás alternativas cubren algún porcentaje de población.

En la Tabla 3 se presentan los resultados de porcentaje de cobertura poblacional según el porcentaje gradiente de ahorro y según el estrato socioeconómico, para cada una de las alternativas analizadas. Se aprecia que la población perteneciente al estrato 3 (medio) registra un porcentaje de ahorro superior a las demás estratificaciones socioeconómicas existentes en cada una de las alternativas propuestas. Por ejemplo, analizando los porcentajes de población cubierta por estrato para un porcentaje gradiente de ahorro de hasta el $20 \%$, se observa que el estrato 3 es el que mayor valor de cobertura refiere para todas las alternativas excepto para la Alternativa Este y para pequeños porcentajes gradiente de ahorro. 
Tabla 2. Porcentaje de cobertura poblacional Vs Gradiente de Ahorro

\begin{tabular}{|c|c|c|c|c|}
\hline $\begin{array}{c}\text { Gradiente de Ahorro de } \\
\text { hasta (\%) }\end{array}$ & $\begin{array}{c}\text { Alternativa } \\
\text { Oeste 1 }\end{array}$ & $\begin{array}{c}\text { Alternativa } \\
\text { Oeste 2 }\end{array}$ & $\begin{array}{c}\text { Alternativa } \\
\text { Norte }\end{array}$ & $\begin{array}{c}\text { Alternativa } \\
\text { Este }\end{array}$ \\
\hline $2,5 \%$ & $86,3 \%$ & $73,0 \%$ & $99,5 \%$ & $46,2 \%$ \\
\hline $5,0 \%$ & $10,0 \%$ & $12,8 \%$ & $9,4 \%$ & $1,3 \%$ \\
\hline $7,5 \%$ & $7,3 \%$ & $3,6 \%$ & $8,0 \%$ & $0,1 \%$ \\
\hline $10,0 \%$ & $4,6 \%$ & $2,4 \%$ & $5,7 \%$ & $0,0 \%$ \\
\hline $12,5 \%$ & $3,4 \%$ & $1,8 \%$ & $3,9 \%$ & $0,0 \%$ \\
\hline $15,0 \%$ & $2,5 \%$ & $1,4 \%$ & $1,0 \%$ & $0,0 \%$ \\
\hline $17,5 \%$ & $1,3 \%$ & $1,0 \%$ & $0,6 \%$ & $0,0 \%$ \\
\hline $20,0 \%$ & $0,7 \%$ & $0,6 \%$ & $0,3 \%$ & $0,0 \%$ \\
\hline $22,5 \%$ & $0,3 \%$ & $0,4 \%$ & $0,1 \%$ & $0,0 \%$ \\
\hline $25,0 \%$ & $0,0 \%$ & $0,2 \%$ & $0,0 \%$ & $0,0 \%$ \\
\hline $27,5 \%$ & $0,0 \%$ & $0,1 \%$ & $0,0 \%$ & $0,0 \%$ \\
\hline $30,0 \%$ & $0,0 \%$ & $0,1 \%$ & $0,0 \%$ & $0,0 \%$ \\
\hline
\end{tabular}

Tabla 3. Cobertura poblacional vs gradiente de ahorro según el estrato socioeconómico, a) Alternativa Oeste 1; b) Alternativa Oeste 2; c) Alternativa Norte; y d) Alternativa Este.

\begin{tabular}{|c|c|c|c|c|c|c|c|c|c|c|c|c|}
\hline \multirow{2}{*}{$\begin{array}{c}\text { G.A. de } \\
\text { hasta (\%) }\end{array}$} & \multicolumn{6}{|c|}{ Alternativa Oeste 1} & \multicolumn{6}{|c|}{ Alternativa Oeste 2} \\
\hline & Est. 1 & Est. 2 & Est. 3 & Est. 4 & Est. 5 & Est. 6 & Est. 1 & Est. 2 & Est. 3 & Est. 4 & Est. 5 & Est. 6 \\
\hline $2,5 \%$ & $96,0 \%$ & $89,4 \%$ & $84,3 \%$ & $95,7 \%$ & $76,3 \%$ & $48,5 \%$ & $95,9 \%$ & $89,3 \%$ & $85,5 \%$ & $97,2 \%$ & $98,9 \%$ & $92,2 \%$ \\
\hline $5,0 \%$ & $4,3 \%$ & $0,1 \%$ & $20,2 \%$ & $1,5 \%$ & $0,0 \%$ & $0,0 \%$ & $7,2 \%$ & $0,3 \%$ & $27,6 \%$ & $25,3 \%$ & $0,0 \%$ & $4,1 \%$ \\
\hline $7,5 \%$ & $2,4 \%$ & $0,0 \%$ & $15,2 \%$ & $0,0 \%$ & $0,0 \%$ & $0,0 \%$ & $3,9 \%$ & $0,0 \%$ & $16,7 \%$ & $0,0 \%$ & $0,0 \%$ & $0,0 \%$ \\
\hline $10,0 \%$ & $0,7 \%$ & $0,0 \%$ & $9,7 \%$ & $0,0 \%$ & $0,0 \%$ & $0,0 \%$ & $1,9 \%$ & $0,0 \%$ & $11,2 \%$ & $0,0 \%$ & $0,0 \%$ & $0,0 \%$ \\
\hline $12,5 \%$ & $0,0 \%$ & $0,0 \%$ & $7,3 \%$ & $0,0 \%$ & $0,0 \%$ & $0,0 \%$ & $0,5 \%$ & $0,0 \%$ & $8,3 \%$ & $0,0 \%$ & $0,0 \%$ & $0,0 \%$ \\
\hline $15,0 \%$ & $0,0 \%$ & $0,0 \%$ & $5,2 \%$ & $0,0 \%$ & $0,0 \%$ & $0,0 \%$ & $0,0 \%$ & $0,0 \%$ & $6,7 \%$ & $0,0 \%$ & $0,0 \%$ & $0,0 \%$ \\
\hline $17,5 \%$ & $0,0 \%$ & $0,0 \%$ & $2,8 \%$ & $0,0 \%$ & $0,0 \%$ & $0,0 \%$ & $0,0 \%$ & $0,0 \%$ & $4,4 \%$ & $0,0 \%$ & $0,0 \%$ & $0,0 \%$ \\
\hline $20,0 \%$ & $0,0 \%$ & $0,0 \%$ & $1,5 \%$ & $0,0 \%$ & $0,0 \%$ & $0,0 \%$ & $0,0 \%$ & $0,0 \%$ & $2,6 \%$ & $0,0 \%$ & $0,0 \%$ & $0,0 \%$ \\
\hline $22,5 \%$ & $0,0 \%$ & $0,0 \%$ & $0,6 \%$ & $0,0 \%$ & $0,0 \%$ & $0,0 \%$ & $0,0 \%$ & $0,0 \%$ & $1,6 \%$ & $0,0 \%$ & $0,0 \%$ & $0,0 \%$ \\
\hline $25,0 \%$ & $0,0 \%$ & $0,0 \%$ & $0,0 \%$ & $0,0 \%$ & $0,0 \%$ & $0,0 \%$ & $0,0 \%$ & $0,0 \%$ & $0,6 \%$ & $0,0 \%$ & $0,0 \%$ & $0,0 \%$ \\
\hline $27,5 \%$ & $0,0 \%$ & $0,0 \%$ & $0,0 \%$ & $0,0 \%$ & $0,0 \%$ & $0,0 \%$ & $0,0 \%$ & $0,0 \%$ & $0,2 \%$ & $0,0 \%$ & $0,0 \%$ & $0,0 \%$ \\
\hline $30,0 \%$ & $0,0 \%$ & $0,0 \%$ & $0,0 \%$ & $0,0 \%$ & $0,0 \%$ & $0,0 \%$ & $0,0 \%$ & $0,0 \%$ & $0,1 \%$ & $0,0 \%$ & $0,0 \%$ & $0,0 \%$ \\
\hline \multirow{2}{*}{$\begin{array}{c}\text { G.A. de } \\
\text { hasta (\%) }\end{array}$} & \multicolumn{6}{|c|}{ Alternativa Norte } & \multicolumn{6}{|c|}{ Alternativa Este } \\
\hline & Est. 1 & Est. 2 & Est. 3 & Est. 4 & Est. 5 & Est. 6 & Est. 1 & Est. 2 & Est. 3 & Est. 4 & Est. 5 & Est. 6 \\
\hline $2,5 \%$ & $99,6 \%$ & $100 \%$ & $99,0 \%$ & $100 \%$ & $100 \%$ & $100 \%$ & $47,8 \%$ & $68,6 \%$ & $29,3 \%$ & $58,6 \%$ & $48,3 \%$ & $57,4 \%$ \\
\hline $5,0 \%$ & $6,2 \%$ & $0,0 \%$ & $19,0 \%$ & $0,0 \%$ & $0,0 \%$ & $0,0 \%$ & $4,7 \%$ & $0,0 \%$ & $2,0 \%$ & $0,0 \%$ & $0,0 \%$ & $0,0 \%$ \\
\hline $7,5 \%$ & $4,3 \%$ & $0,0 \%$ & $16,4 \%$ & $0,0 \%$ & $0,0 \%$ & $0,0 \%$ & $0,0 \%$ & $0,0 \%$ & $0,2 \%$ & $0,0 \%$ & $0,0 \%$ & $0,0 \%$ \\
\hline $10,0 \%$ & $2,9 \%$ & $0,0 \%$ & $11,7 \%$ & $0,0 \%$ & $0,0 \%$ & $0,0 \%$ & $0,0 \%$ & $0,0 \%$ & $0,0 \%$ & $0,0 \%$ & $0,0 \%$ & $0,0 \%$ \\
\hline $12,5 \%$ & $2,1 \%$ & $0,0 \%$ & $7,9 \%$ & $0,0 \%$ & $0,0 \%$ & $0,0 \%$ & $0,0 \%$ & $0,0 \%$ & $0,0 \%$ & $0,0 \%$ & $0,0 \%$ & $0,0 \%$ \\
\hline $15,0 \%$ & $1,6 \%$ & $0,0 \%$ & $2,0 \%$ & $0,0 \%$ & $0,0 \%$ & $0,0 \%$ & $0,0 \%$ & $0,0 \%$ & $0,0 \%$ & $0,0 \%$ & $0,0 \%$ & $0,0 \%$ \\
\hline $17,5 \%$ & $1,1 \%$ & $0,0 \%$ & $1,2 \%$ & $0,0 \%$ & $0,0 \%$ & $0,0 \%$ & $0,0 \%$ & $0,0 \%$ & $0,0 \%$ & $0,0 \%$ & $0,0 \%$ & $0,0 \%$ \\
\hline $20,0 \%$ & $0,6 \%$ & $0,0 \%$ & $0,6 \%$ & $0,0 \%$ & $0,0 \%$ & $0,0 \%$ & $0,0 \%$ & $0,0 \%$ & $0,0 \%$ & $0,0 \%$ & $0,0 \%$ & $0,0 \%$ \\
\hline $22,5 \%$ & $0,1 \%$ & $0,0 \%$ & $0,1 \%$ & $0,0 \%$ & $0,0 \%$ & $0,0 \%$ & $0,0 \%$ & $0,0 \%$ & $0,0 \%$ & $0,0 \%$ & $0,0 \%$ & $0,0 \%$ \\
\hline
\end{tabular}

G.A. Gradiente de Ahorro

De igual forma, en la tabla 4 e presenta el porcentaje gradiente de ahorro de tiempo medio de viaje ponderado por población cubierta según estrato para cada alternativa estudiada; es posible identificar que para todas las alternativas estudiadas excepto para la alternativa Este, es el estrato 3 el que mayores porcentajes de ahorro de tiempo registra, en donde la alternativa Oeste 2 registra el mayor valor de porcentaje de ahorro ponderado por población con un 4,1\%. Analizando cada uno de los estratos de forma individual, se encuentra que la alternativa Norte sería la más conveniente para los estratos 1, 2, 5 y 6; mientras que la alternativa Oeste 2 sería la más conveniente para los estratos 3 y 4 . Es de resaltar que la alternativa más conveniente, de forma general, es la Oeste 2, la cual registra un porcentaje gradiente de ahorro ponderado por población de 3,3\%, para la cual el orden de mayor a menor impacto, se presenta en los estratos 3, 4, 1, 5, 6 y 2, respectivamente.

Se resalta el comportamiento de cobertura poblacional del estrato 1 (bajo), el cual posee un ahorro significativo en las distintas alternativas, y más en la alternativa Norte, traduciéndose esto en una mejora en la conectividad a un porcentaje de habitantes con mayores desventajas a nivel social y económico; no obstante, es necesario resaltar que con el objetivo de aumentar las probabilidades de que exista cada vez mayor equidad en las condiciones de acceso al trabajo, estudio, comercio y demás equipamientos urbanos (Cerda et al., 2012a), se debe fortalecer el sistema de transporte público colectivo urbano, el cual es el modo de transporte que con mayor probabilidad usarían los habitantes de los estratos bajos (1 y 2). Dado lo anterior, el impacto de las alternativas estudiadas no necesariamente registraría iguales beneficios según estrato si se analizan por separado los modos de transporte público y privado. 
Tabla 4. Porcentaje gradiente de ahorro de tiempo medio de viaje ponderado por población cubierta según estrato para cada alternativa estudiada

\begin{tabular}{|c|c|c|c|c|}
\hline Estrato & $\begin{array}{c}\text { Alternativa } \\
\text { Oeste 1 }\end{array}$ & $\begin{array}{c}\text { Alternativa } \\
\text { Oeste 2 }\end{array}$ & $\begin{array}{c}\text { Alternativa } \\
\text { Norte }\end{array}$ & $\begin{array}{c}\text { Alternativa } \\
\text { Este }\end{array}$ \\
\hline 1 & $2,6 \%$ & $2,7 \%$ & $3,0 \%$ & $1,3 \%$ \\
\hline 2 & $2,2 \%$ & $2,2 \%$ & $2,5 \%$ & $1,7 \%$ \\
\hline 3 & $3,7 \%$ & $4,1 \%$ & $3,9 \%$ & $0,8 \%$ \\
\hline 4 & $2,4 \%$ & $3,1 \%$ & $2,5 \%$ & $1,5 \%$ \\
\hline 6 & $1,9 \%$ & $2,5 \%$ & $2,5 \%$ & $1,2 \%$ \\
\hline $\begin{array}{c}\text { Porcentaje Gradiente de Ahorro } \\
\text { ponderado por población total }\end{array}$ & $1,2 \%$ & $2,4 \%$ & $2,5 \%$ & $1,4 \%$ \\
\hline
\end{tabular}

La metodología de evaluación de proyectos de infraestructura vial que aquí se propone, puede ser replicada en otros ciudades y contextos, teniendo como base la red de infraestructura vial, la cual puede ser descargada desde páginas de acceso libre cómo OpenStreetMap.org (https://download.geofabrik.de/) o construida por el equipo de evaluación. Asimismo, los polígonos de población pueden ser construidos a través de datos de acceso abierto suministrados por las entidades locales. Para la calibración de la red y el cálculo del modelo de accesibilidad bastan software de acceso libre como QGIS, permitiendo que está metodología sea utilizada por entidades locales y regionales de una forma fácil y a bajo costo. En definitiva, se considera que la principal aportación científica que se realiza mediante la presente investigación, es la de poder calcular el impacto, en términos de porcentaje gradiente de ahorro, según una distribución de la capacidad económica (estrato socioeconómico) de los habitantes de una ciudad o comunidad, mediante la aplicación de una metodología totalmente replicable y sencilla de ejecutar.

\section{CONCLUSIONES}

Dentro de las opciones aportadas por la administración municipal, se puede observar una correcta ubicación de las alternativas, en cuanto a los sectores de implementación, sin embargo, de las 4 alternativas analizadas, la mejor opción se centra sobre la alternativa Norte, en segundo lugar estaría la alternativa Oeste 1; en éstas, si bien el valor de ahorro no es de gran proporción, la cobertura lograda genera un atractivo importante para estas intervenciones, de igual forma se podría solventar las problemáticas de conexión al permitirle a los usuarios un segundo acceso en caso de alguna eventualidad sobre el corredor actual. Se concluye entonces que los impactos en base a la comparación de las condiciones de accesibilidad, trascienden los territorios en los cuales se realizan directamente las intervenciones infraestructurales, identificándose zonas del área de estudio con cierto grado de afectación, en donde dicha afectación depende de la configuración de la red, del sistema actual de servicios de transporte y de su configuración futura.

La metodología de evaluación permite estimar el impacto sobre la ejecución de las obras, vinculando modelos de cobertura que generalizan el alcance de la intervención; no obstante, se entiende que se deben realizar estudios de pre factibilidad que garanticen una excelente implantación del acceso, de forma segura y con las condiciones operativas eficientes. Las características de conectividad actuales, evidencian la necesidad urgente de la nueva conexión vial, por lo tanto, la administración municipal, debería propiciar las condiciones óptimas para la ejecución de obras en un periodo corto de tiempo, con lo cual se garantizarían una eficiente conexión entre los focos conurbados y una mejora en la calidad de vida de los usuarios del corredor, Finalmente, se debe tener en cuenta la complementariedad del estudio con análisis de demanda a partir de la matriz origen y destino para la ciudad de Manizales, actualizada al año 2017; con lo cual se lograría una mejor interacción entre los modelos de oferta y demanda del transporte, al permitir caracterizar los volúmenes vehiculares que harían uso en la conexión.

\section{AGRADECIMIENTOS}

Los investigadores quisieran agradecer al grupo de investigación en Movilidad Sostenible de la Universidad Nacional de Colombia, particularmente al estudiante de Ingeniería Civil Nicolás Castro, quien hace parte del Semillero de Investigación y colaboró activamente en la creación de las alternativas y su inclusión en el grafo de la red de infraestructura vial del área de estudio.

\section{REFERENCIAS}

Alcaldía de Manizales, Plan de Movilidad de Manizales, Universidad Nacional de Colombia, Manizales (2011)

Alcaldía de Manizales, Plan de Ordenamiento Territorial del Municipio de Manizales 2015-2027, Componente General, Manizales: Alcaldía de Manizales, (2017a)

Alcaldía de Manizales, Plan Maestro de Movilidad: Formulación del Plan maestro de Movilidad de Manizales, Manizales (2017b) 
Cardona, S., Propuesta Metodológica para el Cálculo de las Penalidades por Giro en Modelos de Accesibilidad, Universidad Nacional de Colombia, Sede Manizales, (2018)

Cerda, J., Marmolejo, C. y Roca, J., Modelo de evaluación de la eficiencia energética y ambiental, de la estructura de actividades y la movilidad, de la región metropolitana de Barcelona - Evalúamet. doi:10.5821/ace.v7i19.2565 Architecture, City and Environment, (19), 129-156 (2012a).

Cerda, J., Pérez, C. y Marmolejo, C., Impacto de las grandes actuaciones urbanísticas y de proyectos de transporte en el área metropolitana de Barcelona, http://dx.doi.org/10.5821/ace.v7i20.2579, Architecture, City and Environment, (20), 199$231(2012 b)$.

Chun, Y. y Griffith, D.A., Spatial Statistics and Geostatistics. In Sage. SAGE Publications. (2013)

Dalvi, M.Q. y Martin, K.M., The Measurement of Accessibility: Some Preliminary Results, http://doi.org/10.1007/BF00165245, Transportation, 5(1), 17-42 (1976)

De Smith, M.J., Goodcild, M.F. y Longley, P.A., Geospatial Analysis: A comprehensive guide to Principles Techniques and Software Tools (6 $\left.6^{\text {th }} \mathrm{Ed}\right)(2018)$

Departamento Administrativo Nacional de Estadística - DANE, Proyecciones de población total por sexo y grupos de edad de 0 hasta 80 y más años (2005 - 2020) (2010)

Díaz, M.A., Geoestadística Aplicada, Universidad Autónoma de México, Ciudad de México, (2002)

Dijkstra, E.W., A Note on Two Problems in Connexion With Graphs, Numerical Mathematics, 1(1), $269-271$ (1959)

Escobar, D.A., Montoya, J.A. y Moncada, C.A., Propuesta Metodológica de Análisis para la Inserción de Futuros Nodos Comerciales en la Ciudad de Manizales (Colombia). doi:10.4067/S0718-07642018000200123, Revista Información Tecnológica, 29(2), 123-132 (2018)

Geurs, K.T., De Montis, A. y Reggiani, A., Recent Advances and Applications in Accessibility Modelling, http://doi.org/10.1016/j.compenvurbsys.2014.09.003, Computers, Environment and Urban Systems, 49, $82-85$ (2015)

Geurs, K.T. y Van Eck, J.R., Accessibility Measures: Review and Applications. Evaluation of Accessibility Impacts of LandUse Transportation Scenarios, and Related Social and Economic Impact, RIVM Report 408505 006, National Institute of Public Health and the Environment, Bilthoven (2001)

Hansen, W.G. How Accessibility Shapes Land Use. http://doi.org/10.1080/01944365908978307. Journal of the American Institute of Planners, 25(2), 73-76 (1959)

Ingram, D.R., The Concept of Accessibility: A Search for an Operational Form, http://doi.org/http://dx.doi.org/10.1080/09595237100185131, Regional Studies, 5(2), 101-107 (1971)

Martínez, S., Escobar, D.A. y Tamayo, J.A., Evaluación Comparativa de dos Alternativas de Infraestructura Tipo Túnel a partir de un Análisis de Accesibilidad Urbana. doi:10.4067/S0718-07642017000400018, Rev. Inf. Tecnol., 28(4), 157-168 (2017)

Marvin, S. y Guy, S., Policy Forum: Towards a New Logic of Transport Planning? http://doi.org/10.3828/tpr.70.2.e7856q7168802614, Town Planning Review, 70(2), 139-158 (1999)

Moncada, C.A., Cardona, S. y Escobar, D.A., Saving Travel Time as an Urban Planning Instrument. Case Study: Manizales, Colombia, http://doi.org/10.5539/mas.v12n6p44, Modern Applied Science, 12(6), 44-57 (2018)

Montoya, J.A., Escobar, D.A. y Moncada, C.A., Propuesta de Ubicación de Nuevos Centros Comerciales, Aplicación de un Análisis de Accesibilidad Territorial Urbana, Revista Espacios, 38(51) (2017)

Morris, J.M., Dumble, P.L. y Wigan, M.R., Accessibility Indicators for Transport Planning, http://doi.org/10.1016/01912607(79)90012-8, Transportation Research Part A: General, 13(2), 91-109 (1979)

Oliver, M.A. y Webster, R., Kriging: a Method of Interpolation for Geographical Information Systems, doi:10.1080/02693799008941549. International Journal of Geographical Information Systems, 4(3), 313-332 (1990)

Ortúzar, J. de D. y Willumsen, L.G., Modelling Transport (Fourth Edi), http://doi.org/10.1002/9781119993308 John Wiley \& Sons, Ltd. (1994)

Pirie, G.H., Measuring Accessibility: A Review and Proposal, http://doi.org/10.1068/a110299, Environment and Planning A, 11(3), 299-312 (1979)

Te Brömmelstroet, M. y Bertolini, L., The Role of Transport-related Models in Urban Planning Practice, http://doi.org/10.1080/01441647.2010.541295, Transport Reviews, 31(2), 139-143 (2011)

Van Wee, B., Accessible Accessibility Research Challenges, http://doi.org/10.1016/j.jtrangeo.2015.10.018, Journal of Transport Geography, 51, 9-16 (2016)

Weibull, J.W., An Axiomatic Approach to the measurement of accessibility, http://doi.org/10.1016/0166-0462(76)90031-4, Regional Science and Urban Economics, 6(4), 357-379 (1976)

Xiao, N. GIS Algorithms: Theory and Applications for Geographic Information Science \& Technology, https://doi.org/10.4135/9781473921498, In Sage. SAGE Publications. (2016)

Zhang, D., y Wang, X.C., Transit Ridership Estimation With Network Kriging: A case study of Second Avenue Subway, NYC. doi:10.1016/j.jtrangeo.2014.08.021. Journal of Transport Geography, 41, 107-115 (2014) 
